\title{
Activités in vitro de plusieurs huiles essentielles sur Bacillus larvae White et essai au rucher
}

\author{
I Floris ${ }^{1}, \mathrm{C}$ Carta ${ }^{2}$, MDL Moretti 3 \\ 1 Istituto di Entomologia agraria; \\ 2 Istituto di Patologia vegetale, Università degli Studi, Via De Nicola; \\ 3 Dipartimento di Scienze farmaceutiche, Università degli Studi, Via Muroni 23/a, 07100 Sassari, Italie
}

(Reçu le 4 mars 1996; accepté le 25 mars 1996)

\begin{abstract}
Résumé - L'activité antibactérienne de plusieurs huiles essentielles sur les formes végétatives et sporulées de six souches de Bacillus larvae White, agent de la loque américaine, a été évaluée in vitro. Les résultats indiquent que les huiles d'oranger (Citrus sinensis), cannelle (Cinnamomum sp), cumin (Cuminum cyminum), giroflier (Eugenia spp), thym (Thymus vulgaris) et verveine (Verbena) présentent in vitro des pouvoirs bactéricides et sporicides ; celle de la cannelle a montré la meilleure activité, avec une concentration minimale bactéricide de $50 \mathrm{ppm}$ et sporicide de $100 \mathrm{ppm}$. À la concentration de $400 \mathrm{ppm}$, incorporée dans le candi, cette huile a été efficace aussi contre la loque américaine dans une expérience en rucher. L'aromathérapie peut donc permettre de contrôler la loque américaine sans avoir recours ni aux sulfamides ni aux antibiotiques et de préserver le caractère naturel du miel.
\end{abstract}

Bacillus larvae / activité antibactérienne / huiles essentielles / aromathérapie / loque américaine

\section{INTRODUCTION}

Les activités antibactérienne et antifongique des huiles essentielles sont depuis longtemps connues en phytothérapie (Maruzzella et al, 1963). Récemment, elles ont été reconnues aussi sur Ascosphaera apis, agent du couvain plâtré (Colin et al, 1989), et sur Bacillus larvae (Carta et Floris, 1989 ; Floris et Carta, 1990 ; Calderone et Shimanuki, 1994), agent de la loque américaine. II faut souligner en outre que l'aromathérapie est pratiquée aussi bien en médecine vétérinaire qu'en médecine humaine (Allegrini et al, 1974 ; Belaiche, 1979). L'utilisation de ces substances naturelles en apiculture présente un grand intérêt par rapport aux méthodes traditionnelles, car elle permet d'éviter le risque de résidus dangereux pour le consommateur comme cela s'est produit avec l'utilisation des antibiotiques (Gilliam et Argauer, 1981 ; Lodesani et al, 1994) et le développement de souches résistantes (Alippi, 1994). En outre, les antibiotiques et 
les sulfamides utilisés dans la pratique apicole agissent seulement par un effet bactériostatique contre Bacillus larvae, donc il est nécessaire de trouver des substances réunissant des pouvoirs bactéricide et sporicide. Pour ces raisons, on a vérifié, d'abord in vitro (Carta et Floris, 1989) et puis dans les ruchers (Floris et Carta,1990), l'action de plusieurs huiles essentielles disponibles dans le commerce en Italie.

\section{MATÉRIEL ET MÉTHODES}

\section{Mesure in vitro des activités}

Les essais in vitro ont été faits sur les huiles suivantes, pour lesquelles on a indiqué la source botanique principale et le type : anis étoile (IIIicium verum Hook, F, E-ANIV/O1), basilic (Ocimum basilicum L, E-BASI/01), bergamotier (Citrus bergamia Risso, E-BERG/01), cumin (Cuminum cyminum L, E-CUM/01), eucalyptus (Eucalyptus globulus Labille, E-EUC/01), genévrier (Juniperus communis L, E-GEN/01), giroflier (Eugenia spp, $\mathrm{E}-\mathrm{GIR} / 01$ ), géranium (Pelargonium graveolans L, E-GER/01), hysope (Hyssopus officinalis L, EHYS/01), lavande (Lavandula officinalis Chaix, E-LVD/01), citron (Citrus limon (L) Burm F, EMELV/01), menthe (Mentha anvensis L, var piperascens, E-MTHS/02), oranger (Citrus sinensis $\mathrm{L}$, E-ORD/01), origan (Thymus vulgaris L, EORI/01), pin (Pinus sp, E-PIN/01), romarin (Rosmarinus officinalis L, E-ROM/01), sarriette (Thymus vulgaris L, E-SAR/01), sauge (Salvia officinalis $L, E-S A G / 01$ ), thym (Thymus vulgaris $L$, $E-T H Y / 01$ ) et verveine (huile reconstituée, EVEIR/01). En outre on a utilisé, in vitro et in vivo, l'huile de cannelle (Cinnamomum sp) dont le tableau I montre la composition chimique déterminée à travers la méthode reportée sur «British Pharmacopoeia" (1995, vol II, Appendix XK) et par chromatographie en phase gazeuse (Peana et al, 1994).

À partir de larves atteintes de loque américaine, provenant de trois ruchers différents de la Sardaigne du Nord, on a isolé trois souches de B larvae (C1, C2, C3), trois autres ont été fournies par l'Institut zooprophylactique de Sassari (C4) et par l'Institut national d'apiculture de Bologna $(\mathrm{C} 5, \mathrm{C} 6)$.
Préalablement l'activité antibactérienne des huiles soit pures soit faiblement diluées (1:5 dans l'éthanol à $95 \%$ ) a été évaluée par la méthode de diffusion en agar (Carta et al, 1989). Les cultures et les essais ont été effectués sur le substrat décrit par Forster et al (1950). Les spores ont été obtenues sur le milieu 'Brain Heart Infusion' de la Difco. Pour chaque souche, une suspension dense dans l'eau a été obtenue à partir d'une culture de 24 heures. Cette suspension, diluée à $1 / 10$, a été utilisée pour ensemencer l'agar nutritif ( $1 \mathrm{~mL}$ suspension / $100 \mathrm{~mL}$ substrat) maintenu en bain thermostatique à $47^{\circ} \mathrm{C}$. Ensuite, le substrat a été coulé dans des boîtes de Pétri de $90 \mathrm{~mm}$; après la solidification, au centre de chaque plaque on a mis un petit disque de papier filtre stérile ('Bacto Concentration Disks' de la Difco) saturé avec les substances à tester; dans les boîtes témoin le disque était imbibé d'éthanol à $95 \%$. Pour chaque souche et pour chaque

Tableau I. Composition de l'huile essentielle de Cinnamomun $\mathrm{sp}$ utilisée dans l'expérience de terrain.

\begin{tabular}{llr}
$N^{\circ}$ & Composant & \multicolumn{2}{c}{ Pourcentage } \\
- & - & - \\
1 & a-theyène & - \\
2 & a-pinène & 1,1 \\
3 & camphène & 1,8 \\
4 & b-pinène & 0,2 \\
5 & phellandrène & 0,1 \\
6 & limonène & 0,1 \\
7 & p-cymène & 0,2 \\
8 & camphre & 0,2 \\
9 & benzaldéhyde & 0,1 \\
10 & linalol & 0,2 \\
11 & bornylacétate & 0,5 \\
12 & b-caryophyllène & 0,5 \\
13 & a-terpinéol & 0,1 \\
14 & bornéol & 0,2 \\
15 & isobutyrate de linalyl & 0,2 \\
16 & géraniol & 0,1 \\
17 & alcool cinnamique & 0,2 \\
18 & aldéhyde cinnamique & 0,5 \\
19 & aldéhyde hydroxycinnamique & 79,3 \\
20 & inconnu & 1,8 \\
21 & eugénol & 0,3 \\
22 & méthyl eugénol & 11,9 \\
23 & inconnu & 0,1 \\
24 & farnésol & 0,2 \\
& & 0,1
\end{tabular}


substance on a effectué trois répétitions. Après 48 heures d'incubation à la température de $36^{\circ} \mathrm{C}$, les huiles qui avaient généré un diamètre d'inhibition d'au moins $20 \mathrm{~mm}$ ont été essayées à différentes concentrations (de 50 à 400 ppm) pour établir les concentrations minimales inhibitrice (CMI), bactéricide (CMB) et sporicide (CMS).

\section{Tolérance}

Pour estimer les effets secondaires sur les abeilles (Apis mellifera ligustica $\mathrm{L}$ ), on a utilisé des cagettes contenant chacune un fragment de rayon et 50 abeilles adultes prélevées dans les ruchers. Pour chaque huile nous avons utilisé trois cagettes. On a décidé d'essayer les effets seulement sur les abeilles adultes en considérant le type d'administration de 'huile choisi pour l'essai in vivo (par le candi). Les différentes huiles essentielles ont été administrées à la concentration de 400 ppm dans du sirop de sucre pendant dix jours. Pendant les expériences, on a enregistré la mortalité des abeilles et le taux de consommation des solutions.

\section{Essais thérapeutiques}

En ce qui concerne les essais sur le terrain, qui ont un caractère préliminaire, un nombre limité de ruchers a été employé en considérant les difficultés pratiques et la nécessité d'avoir à disposition des groupes de colonies génétiquement homogènes. Dans le premier essai, on a utilisé seulement l'huile qui s'est révélée la plus efficace in vitro, c'est-à-dire l'huile de cannelie. Les effets de cette huile sur l'évolution de la loque américaine ont été jugés sur 4 nuclei, provenant de la même ruche, ayant de jeunes reines fécondées et approximativement des quantités égales de couvain, miel et pollen (Lindenfelser, 1968). L'infection a été réalisée par l'aspersion de la surface du couvain d'une suspension aqueuse contenant environ $1 \times 10^{8} \mathrm{~mL}$ de spores de $B$ larvae. L'huile a été administrée (d'octobre à décembre) à la concentration de $400 \mathrm{ppm}$ incorporée dans un candi au miel (1 partie de miel/1,5 partie de sucre glace). La dose par colonie traitée était de $1,2 \mathrm{~g}$ d'huile, répartie dans $3 \mathrm{~kg}$ de candi et administrée en six doses hebdomadaires de $0,5 \mathrm{~kg}$ chacune. Trois ruches ont été nourries avec le candi aromatisé : la première au moment de l'infection (début octobre), la deuxième 10 jours après et la troisième 20 jours après l'infection. La ruche témoin a reçu le candi simple.

Dans le deuxième essai, cinq colonies ont été obtenues comme indiqué ci-dessus : deux ont été traitées avec le candi aromatisé ; deux par le sulfathiazol (Giordani et al, 1982) et une avec du candi simple. L'essai a commencé en août lorsque les symptômes de la loque américaine étaient évidents.

Au cours des deux essais on a examiné périodiquement (toutes les 2 semaines) le couvain pour évaluer l'évolution de la maladie, en observant l'état général du couvain et en prélevant un échantillon d'environ 30 larves pour l'analyse de laboratoire (Lloyd, 1986).

\section{RÉSULTATS}

\section{Essais in vitro (tableaux II, II et IV; fig 1)}

Pures, la plupart des huiles montre une action inhibitrice sur toutes les souches de $B$ larvae. À la dilution de $1 / 5$, l'efficacité des huiles de cannelle, cumin et verveine est presque inchangée, réduite pour celles de bergamotier, giroflier, géranium, hysope, citron, menthe, oranger, romarin, sarriette et thym, négligeable pour les autres : anis, basilic, eucalyptus, genévrier, lavande, origan, pin et sauge. Les essais à différentes concentrations indiquent que l'huile de cannelle est la plus efficace (CMB $=50-100$ ppm ; CMS $=100-200$ ppm). Dans tous les cas, la CMB a été au maximum de 200 ppm et la CMS de 300 ppm.

L'analyse de variance a été utilisée pour évaluer les différences dans l'action des huiles essentielles sur les six souches de $B$ larvae.

\section{Tolérance}

Les ouvrières des cagettes ont accepté de la même façon les différentes solutions 


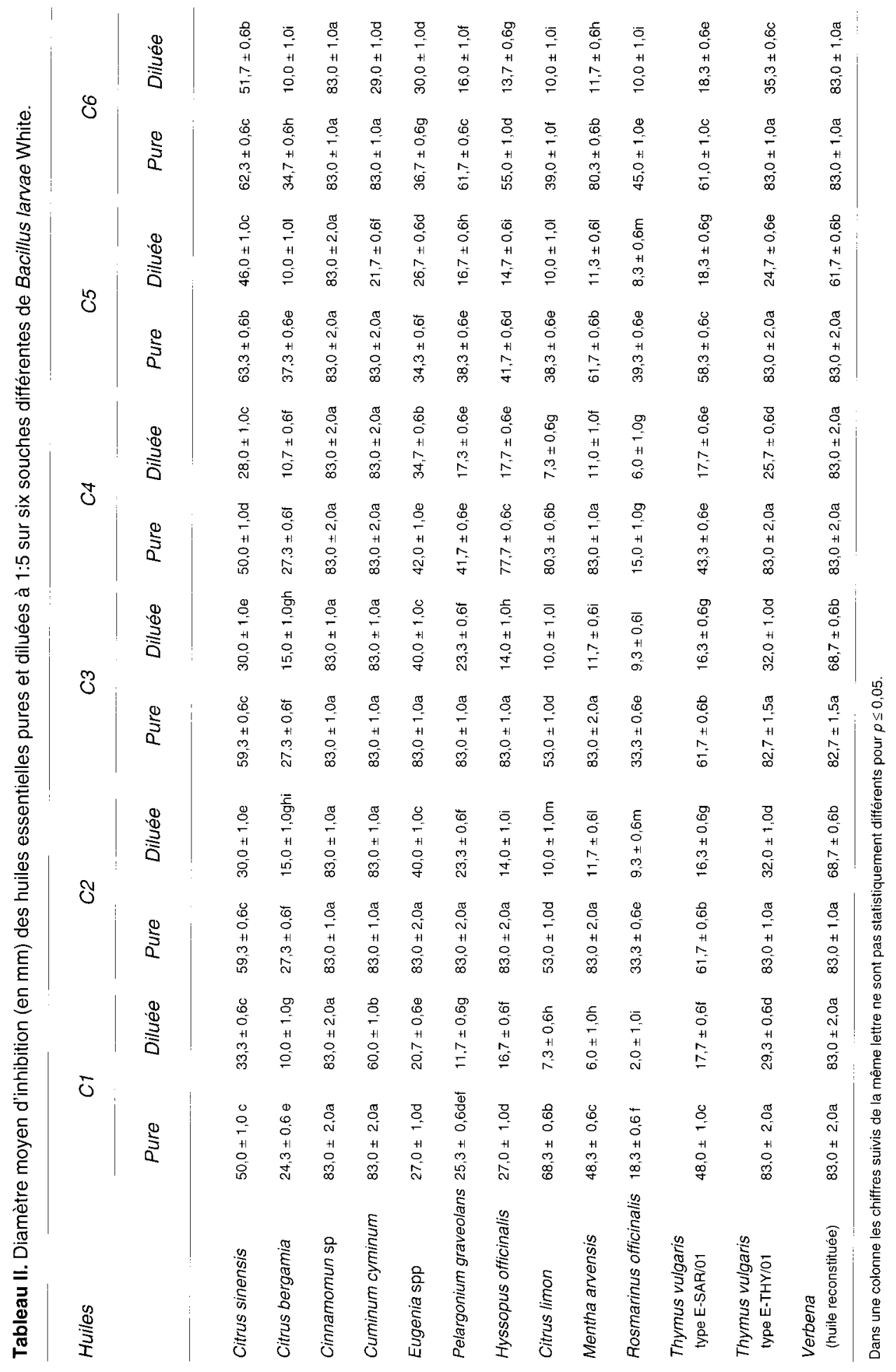


d'huiles essentielles administrées : en moyenne 50 abeilles avaient consommé $60,1 \pm 8,9 \mathrm{mg}$ de solution en dix jours avec une mortalité de $28,7 \pm 6,2 \%$ abeilles. Dans les cagettes témoin 50 abeilles avaient consommé $78,2 \pm 22,5 \mathrm{mg}$ de sirop de sucre pour une mortalité de $24 \pm 7,2 \%$. L'analyse statistique n'indique pas de différence significative au seuil de $5 \%$ parmi les cagettes autant pour la mortalité des abeilles que pour la quantité de sirop consommé.

\section{Essais de terrain}

Sur le terrain, le traitement à l'huile de cannelle s'est révélé efficace en prévention. À l'examen effectué deux semaines après le début du traitement on a trouvé des larves qui présentaient les symptômes caractéristiques de la loque américaine dans trois des quatre colonies, à l'exception de celle traitée au moment de l'aspersion des spores. Après 4 semaines d'administration du candi

Tableau III. Concentration (mg/kg) minimale bactéricide (CMB) des huiles essentielles les plus efficaces sur six souches différentes de Bacillus larvae White.

Huiles

C1
C2

100

100

100

200

200

Thymus vulgaris

(type E-THY/01) 200

Verbena

(huile reconstituée)

200

$\begin{array}{rr}100 & 200 \\ 50 & 100 \\ 100 & 100 \\ 100 & 200 \\ 200 & 200 \\ 200 & 200\end{array}$

\section{Souches}

$\begin{array}{lcccr}\text { C3 } & \text { C4 } & \text { C5 } & \text { C6 } & \begin{array}{l}\text { Moyenne } \\ \pm \text { écart type }\end{array} \\ & & & & \\ 200 & 200 & 100 & 100 & 133,3 \pm 51,6 \\ 100 & 100 & 100 & 100 & 91,7 \pm 20,4 \\ 100 & 200 & 100 & 200 & 133,3 \pm 51,6 \\ 200 & 200 & 200 & 200 & 183,3 \pm 40,8 \\ 200 & 200 & 200 & 200 & 200,0 \pm 0,0 \\ 200 & 200 & 200 & 100 & 183,3 \pm 40,8\end{array}$

Tableau IV. Concentration (mg/kg) minimale sporicide (CMS) des huiles essentielles les plus efficaces sur six souches differentes de Bacillus larvae White.

Huiles

Souches

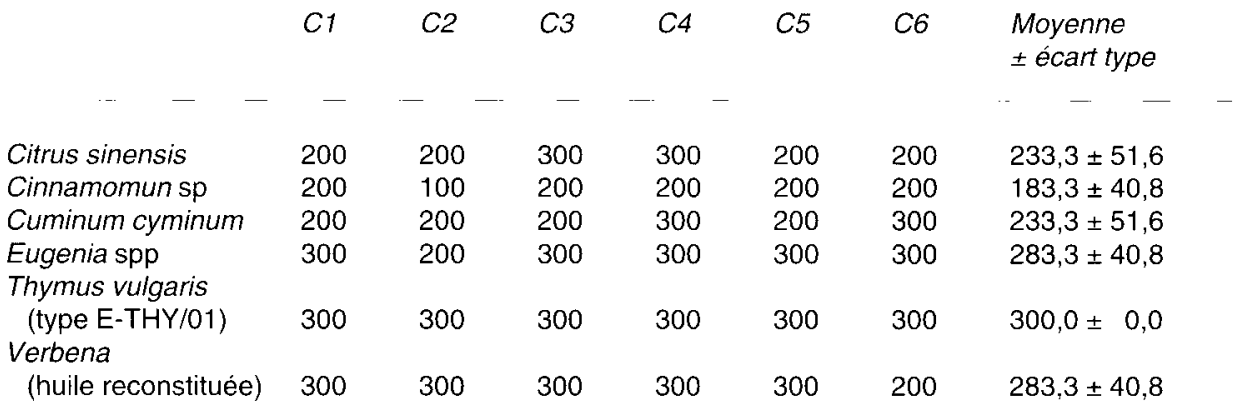



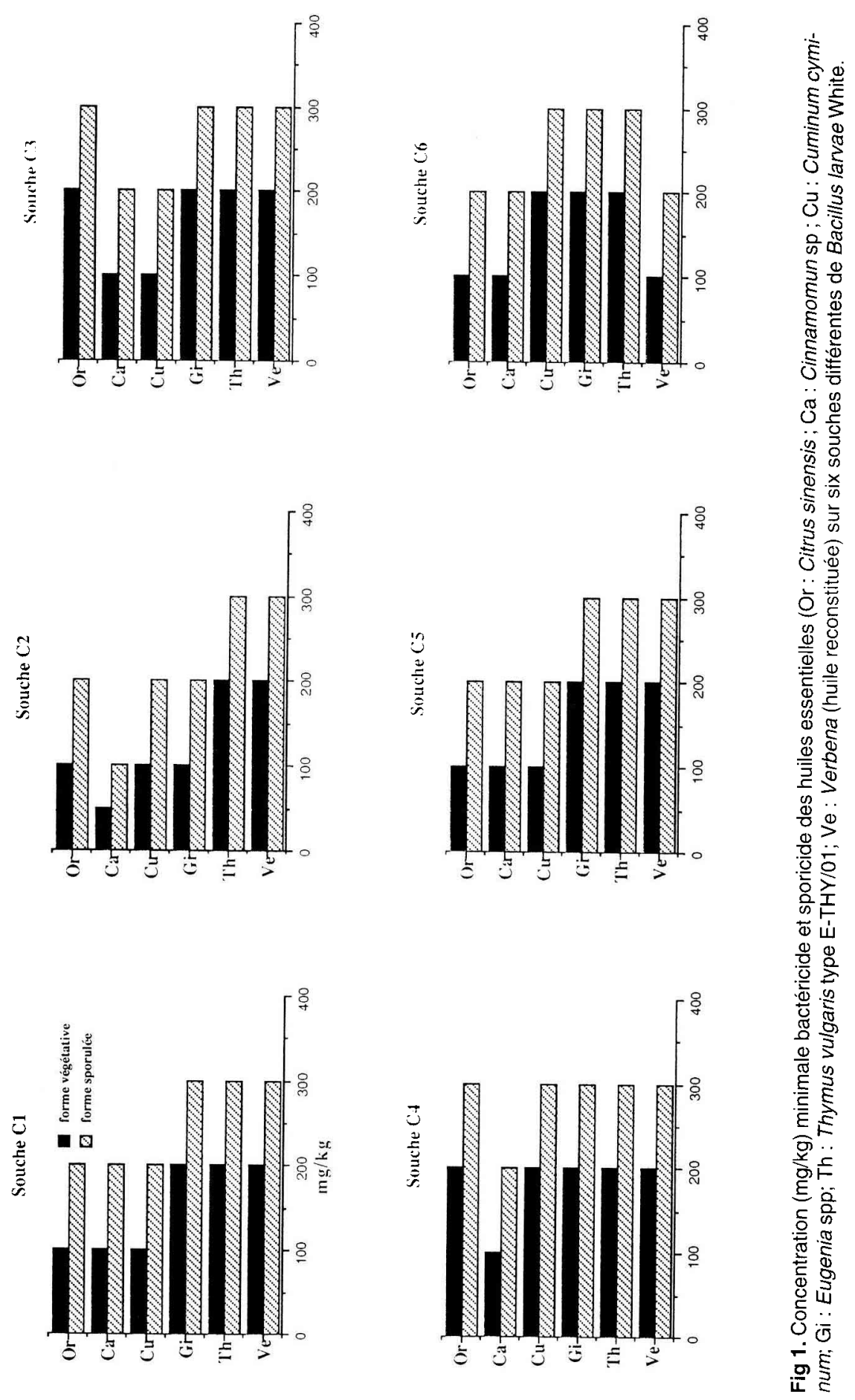
aromatisé, à l'observation du couvain sur le terrain et au laboratoire, les colonies traitées apparaissaient entièrement guéries et seule la colonie témoin restait atteinte. Au début de l'été (juin), les symptômes visibles sont réapparus aussi sur les colonies traitées.

Dans la deuxième expérience, le sulfathiazol comme le candi aromatisé a provoqué une interruption temporaire de l'évolution de la loque.

\section{DISCUSSION ET CONCLUSIONS}

L'aspect le plus important à souligner est l'activité bactéricide et sporicide in vitro de plusieurs huiles essentielles. II faudrait mieux utiliser ces activités pour le contrôle de la loque américaine. Nos expériences ont confirmé la forte activité antibactérienne in vitro de l'huile de Cannelle. En ce qui concerne le terrain, les essais effectués confortent les résultats de laboratoire, en particulier pour la période automne-hiver, mais nécessitent une confirmation sur une base expérimentale plus vaste. Dans ce cas, I'huile pourrait remplacer le sulfathiazol et vraisemblablement les antibiotiques utilisés dans le contrôle de la maladie surtout pendant les traitements préventifs hivernaux. Cependant, l'administration choisie de l'huile dans les ruchers (par la nourriture) ne peut pas être considérée comme une désinfection, donc le problème de l'élimination de tout le matériel infecté par les spores demeure entier.

Les recherches futures devraient établir d'autres méthodes plus appropriées d'utilisation des huiles aussi pendant le printemps et pendant l'été, complétées par des techniques apicoles comme, par exemple, la réduction à l'état d'essaim, qui assure l'élimination de la plupart des spores dans la ruche, permettant ainsi d'obtenir une amélioration radicale de l'état de santé des colonies atteintes de loque américaine.

\section{REMERCIEMENTS}

Les auteurs remercient les docteurs M Dettori et A Lentini pour leur collaboration.

\section{Summary - Activity of various essen- tial oils against Bacillus larvae White in vitro and in apiary trials. The action of 21} essential oils against six strains of Bacillus larvae White, the causal agent of American foulbrood of honeybees, was studied in vitro. In a preliminary trial (table II) the effectiveness of either pure or diluted (1:5) oils was assessed by the diffusion agar method (Carta et al, 1989). The more effective ones were then tested in nutrient broth, at doses ranging from $50-400 \mathrm{mg} / \mathrm{kg}$, to determine the minimal bactericide and sporicide concentrations. The most significant results were obtained with the oils of Citrus sinensis, Cinnamomun sp, Cuminum cyminum, Eugenia spp, Thymus vulgaris and reconstituted oil of Verbena (tables III and IV, fig 1). The same oils proved to be non-toxic for adult honeybees at the dose of $400 \mathrm{mg} / \mathrm{kg}$. Cinnamomun oil was the most effective, with minimal bactericide and sporicide concentrations of 50 and $100 \mathrm{mg} / \mathrm{kg}$ respectively. This oil also gave positive results in controlling the American foulbrood in field trials, particularly in autumn and winter, when administered in semi-solid food (honey + caster sugar, 1:1.5) at a concentration of $400 \mathrm{mg} / \mathrm{kg}$. In particular, the action of Cinnamomun oil on disease growth was evaluated in two distinct trials using four and five nuclei, respectively, obtained from the same colony (Apis mellifera ligustica Spin) so that each of them contained an approximately equal amount of adult bees, brood, honey and pollen beside a young queen (Liendenfelser, 1968). In both experimental trials, the worker brood was also carefully examined periodically by laboratory tests (Lloyd, 1986). The results showed that during autumn and winter, when the bees' 
consumption of the extra food is favoured by the environmental conditions (weather, scarcity or absence of flowers), Cinnamomun oil acts also in vivo on $B$ larvae. However, its use in the control of American foulbrood poses several practical problems including the time and the method of administration to take full advantage of the bactericidal properties found in vitro. If essential oils can be used against American foulbrood, the toxicological risks and the establishment of undesirable resistance factors associated with use of chemicals would be avoided.

\section{Bacillus larvae /antimicrobial action / essential oil / American foulbrood control}

\section{Zusammenfassung - Wirksamkeit ver- schiedener ätherischer Öle gegen Bacil-} lus larvae White in vitro und Versuche am Bienenstand. Die Wirksamkeit von 21 ätherischen Ölen gegen 6 Linien von Bacillus larvae White, dem Erreger der amerikanischen Faulbrut bei Honigbienen, wurde in vitro untersucht. In Vorversuchen (Tabelle II) wurde die Wirksamkeit von reinen Ölen sowie ihrer vedünnten Lösungen (1:5) mit der "Diffusion Agar Methode" (Carta et al, 1989) bestimmt. Anschließend wurden nur die wirksamsten weiter in Futterlösung (nutrient broth) untersucht. Um die kleinsten bakterizide und sporozide Konzentrationen zu bestimmen, wurde die Dosis von 50 bis 400 $\mathrm{mg} / \mathrm{kg}$ variiert. Die deutlichsten Ergebnisse wurden mit Ölen von Citrus sinensis, Cinnamomun sp, Cuminum cyminum, Eugenia spp, Thymus vulgaris und wieder aufbereitetem Öl von Verbena (Tabelle III und IV, Abb 1) erzielt. Bei der Dosierung von 400 $\mathrm{mg} / \mathrm{kg}$ war dieses Öl für adulte Bienen ungiftig. Cinnamomum Öl zeigte die größte Wirksamkeit, die minimale bakterizide bzw sporozide Konzentration betrug 50 und 100 $\mathrm{mg} / 1 \mathrm{~kg}$. Dieses Öl zeigte auch eine positive Wirkung bei der Behandlung der amerikanischen Faulbrut in Feldversuchen, beson- ders im Herbst und Winter, wenn es mit Futterteig (Honig + Puderzucker, 1:1,5) in einer Dosis von $400 \mathrm{mg} / \mathrm{kg}$ verabreicht wird. Weiterhin wurde die Wirksamkeit von Cinnamomun Öl in 2 getrennten Versuchen bestimmt: 4 bzw 5 Ableger wurden jeweils aus dem gleichen Volk gebildet (Apis mellifera ligustica Spin), sodaß alle etwa die gleiche Anzahl adulter Bienen, Brut, Honig und Pollen und eine junge Königin (Lindenfelser, 1968) erhielten. In beiden Versuchen wurde die Brut in festen Zeitabständen mit Labortests untersucht (Lloyd, 1986). Die Ergebnisse zeigten, daß Cinnamomun Öl in vivo im Bienenvolk eine Wirkung hat, wenn im Herbst und im Winter, bedingt durch die Außenbedingungen (Wetter, nur wenige oder gar keine Blüten), die Aufnahme von Zusatzfutter durch die Bienen begünstigt wird. Die Nutzung des Öls für die Behandlung der amerikanischen Faulbrut mit der vollen Wirksamkeit der in vitro gefundenen bakteriziden Eigenschaften wirft jedoch einige praktische Probleme auf, die sowohl die Zeit als auch die Methode der Applikation betreffen. Der Einsatz ätherischer Öle gegen amerikanische Faulbrut könnte möglicherweise toxikologische Risiken und die Bildung unerwünschter Resistenzen, verursacht durch wahllose Anwendung von Chemikalien, vermeiden.

\section{Bacillus larvae / antimicrobial Wirkung/ ätherische Öle / Behandlung der ameri- kanischen Faulbrut}

\section{RÉFÉRENCES}

Alippi AM (1994) In vitro sensitivity of Bacillus larvae to different antibiotics. Vida Apícola 66, 20-24

Allegrini J, Simeon de Buochberg M, Pellecuer J (1974) Étude in vitro de l'activité antibactérienne et antifongique de l'essence de Satureia montana L. J Pharm Belg 29, 137-144

Belaiche P (1979) Traité de phytothérapie et d'aromathérapie. Tome 1I. Maladies infectieuses. Maloine, Paris 
Calderone NW, Shimanuki $H$ (1994) An in vitro evaluation of botanical compounds for the control of the honeybee pathogens Bacillus larvae and Ascosphaera apis, and the secondary invader $B$ alvei. J Essent Oil Res 6, 279-287

Carta C, Floris I (1989) Prospettive di controllo delia peste americana delle api con oli essenziali. Prove preliminari. Società Italiana Ecologia, Atti 8, 183-187

Carta C, Foddai A, Marras F (1989) Attività di oli essenziali e di alcuni loro costituenti su Agrobacterium tumefaciens (Smith et Townsend) Conn biotipo 1 Keane et al. Atti Giornate Fitopatologiche 1, 527-536

Colin ME, Ducos de Lahitte J, Larribau E, Boué T (1989) Activité des huiles essentielles de labiées sur Ascosphaera apis et traitement d'un rucher. Apidologie $20,22 \uparrow-228$

Floris I, Carta $C(1990)$ In vivo activity of Cinnamomum zeylanicum Nees essential oil against Bacillus larvae White. Apicoltura 6, 57-61

Forster G, Hardwick WA, Guirard B (1950) Antisporulation factors in complex organic media. I. Growth and sporulation studies of Bacillus larvae. J Bact 59 , 463-470
Gilliam M, Argauer RJ (1981) Oxytetracycline residues in surplus honey, brood nest honey and larvae after medication of colonies of honey bees, Apis mellifera, with antibiotic extender patties, sugar dusts, and syrup sprays. Environ Entomol 10, 479-482

Giordani G, Vecchi MA, Nardi M (1982) Nozioni pratiche sulle malattie delle api. Federazione Apicoltori Italiani ed, Roma, 23-44

Lindenfelser LA (1968) In vivo activity of propolis against Bacillus larvae. J invertebr Pathol 12, 129-131

Lodesani M, Carpana E, Bassini A, Dottori M, Mascher A, Lavazza A (1994) Ricerca di residui di ossitetraciclina in alveari trattati secondo due diversi metodi di somministrazione. Apicoltura 9, 51-66

Lloyd JM (1986) Simplified laboratory diagnosis of American foulbrood disease. J Apic Res 25, 55-57

Maruzzella JC, Reine S, Solat H, Zeitlin H (1963) The action of essential oils on phytopathogenic bacteria. Plant Disease Reporter 47, 23-26

Peana A, Satta M, Moretti MDL, Orecchioni M (1994) A study on choleretic activity of Salvia desoleana essential oil. Planta Med 60, 478-479 